\title{
Non-Convulsive Status Epilepticus
}

National Cancer Institute

\section{Source}

National Cancer Institute. Non-Convulsive Status Epilepticus. NCI Thesaurus. Code C155291.

Status epilepticus without prominent motor symptoms, the duration of which is at least ten minutes. 